\title{
Diffraction imaging and tomography investigations on the hydration of gypsum plaster
}

\author{
M. La Bella ${ }^{1,2}$, R. Besselink ${ }^{2}$, A. E. S. Van Driessche ${ }^{2}$, J. P. Wright ${ }^{1}$, C. Giacobbe ${ }^{1}$, A. Fernandez-Martinez ${ }^{2}$ \\ ${ }^{1}$ European Synchrotron Radiation Facility, 71 Avenue Des Martyrs, Grenoble 38040, France, \\ ${ }^{2}$ Institut de science de la Terre, Université Grenoble Alpes, 1381 Rue de la Piscine, Gières 38610, France
}

michela.la-bella@esrf.fr

The three-dimensional X-ray diffraction (3dxrd) technique provides a useful tool to investigate polycrystalline materials, grain-bygrain, in a non-destructive way. The approach of the scanning $3 \mathrm{dxrd}$ microscopy is to probe the sample by moving a pencil beam horizontally across it (y direction) with a resolution dependent on the beam size. For each step, the sample is rotated of $180^{\circ}$ (or $360^{\circ}$, $\omega$ angle) in order to collect the diffraction spots of all the grains in the sample [1].

We used a combined approach of scanning 3dxrd and Phase Contrast Tomography (PCT) to investigate the hydration of a widespread hydraulic binder material, namely gypsum plaster. This material forms when the bassanite (calcium sulfate hemihydrate) reacts with water. In-situ 3dxrd measurements allowed to understand the crystallographic lattice, orientation and position of each grain in the sample during the hydration reaction (Figure $1 \mathrm{a}, \mathrm{b}$ ).

The PCT reconstructions, instead, allowed the visualization of the shape of the crystals in the sample over time and a quantification of density and porosity (Figure $1 \mathrm{c}, \mathrm{d}$ ).

Monitoring the evolution of the hydration reaction of gypsum plaster with both these techniques appears to be a promising tool to gain insights about the kinetics of the hydration reaction, the crystallization and growth of the hydrated phase and the shape of the final gypsum crystals that build the interlocked and porous gypsum plaster hardened mass.

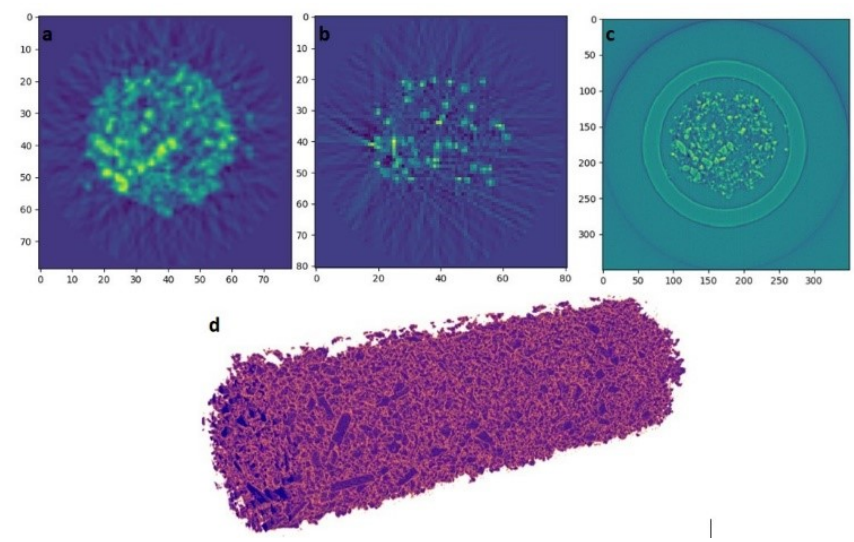

Figure 1. From the left: a) reconstruction of the grains of the sample from the $3 \mathrm{dxrd}$ diffraction intensity; b) preliminary grain map showing some indexed grains; c) PCT slice showing the shape of the crystals; d) PCT reconstruction of the volume of the sample.

[1] Hayashi, Y., Hirose, Y., Seno, Y. (2015). J. Appl. Cryst. 48, 1094-1101.

Keywords: Materials science; diffraction; gypsum; grains 